\title{
INFLUENCE OF BIAS POTENTIAL AND SUBSTRATE ORIENTATION ON CHARACTERISTICS OF DEPOSITED COATING: THE ROLE OF SPUTTERING
}

\author{
A.I. Kalinichenko, E.N. Reshetnyak, V.E. Strel'nitskij \\ NSC "Kharkiv Institute of Physics and Technology" NASU \\ Kharkiv, 61108, st. Akamemicheskaya 1, Ukraine \\ E-mail: aikalinichenko@kipt.kharkov.ua \\ Received 23 March, 2018
}

Influence of atomic sputtering on intrinsic stress and growth rate of the coating made by method of plasma - ion deposition with use of pulsed bias potential and at different incidence angles of ions is theoretically investigated. The formula for intrinsic stress calculation in deposited coating obtained in the model of the nonlocal thermoelastic peak of the ion, taking into account atomic sputtering processes, was used to calculate the stresses in TiN and $\mathrm{CrN}$ coatings deposited from $\mathrm{Ti}^{+}$and $\mathrm{Cr}^{+}$ion beams, respectively. The stress value for the coatings considered correlates with the modulus of elasticity of the coating material. The stress curve maximum decreases and shifts to the region of higher potentials with increasing angle of incidence. This behavior is due to the sputtering of quasi-stable interstitial defects that determine the level of stress in the deposited coating. A formula is proposed for the coating deposition rate, which takes into account the sputtering of the coating atoms at arbitrary bias potential and the angle of incidence of the ions. It is shown that sputtering sharply reduces the coatings deposition rate and makes it impossible to deposit TiN and $\mathrm{CrN}$ coatings in the DC mode at potentials on the substrate exceeding $1.7 \mathrm{kV}$ and $0.7 \mathrm{kV}$, respectively, and with normal ion incidence. Sputtering has the greatest influence on the intrinsic stress and the growth rate of the coating at ion deposition at inclined angles of incidence $\alpha=45^{\circ} \ldots 70^{\circ}$. The results of the calculations are compared with the available experimental data.

KEY WORDS: plasma-ion coating deposition, pulsed bias potential, intrinsic stress, non-local thermoelastic peak of ion, deposition rate.

\section{ВЛИЯНИЕ ПАРАМЕТРОВ ПОТЕНЦИАЛА СМЕЩЕНИЯ И ОРИЕНТАЦИИ ПОДЛОЖКИ НА ХАРАКТЕРИСТИКИ ОСАЖДАЕМОГО ПОКРЫТИЯ: РОЛЬ РАСПЫЛЕНИЯ}

А.И. Калиниченко, Е.Н. Решетняк, В.Е. Стрельницкий

Национальный Научный Центр «Харьковский физико-технический институт» 61108, Харьков, Академическая, 1

Теоретически исследуется влияние процессов атомного распыления на величину внутренних напряжений и скорость роста покрытия, получаемого методом плазменно-ионного осаждения с использованием импульсного потенциала смещения на подложке и при различных углах падения ионов на осаждаемую поверхность. Формула для расчета внутренних напряжений в осаждаемом покрытии, полученная в рамках модели нелокального термоупругого пика иона с учетом процессов атомного распыления, использовалась для расчета напряжений в покрытиях $\mathrm{TiN}$ и $\mathrm{CrN}$, осаждаемых из потоков ионов $\mathrm{Ti}^{+}$и $\mathrm{Cr}^{+}$, соответственно. Величина напряжений для рассмотренных покрытий коррелирует с модулем упругости материала покрытия. Максимум кривой напряжений уменьшается и смещается в область более высоких потенциалов с ростом угла падения. Такое поведение обусловлено процессом распыления квазистабильных междоузельных дефектов, определяющих уровень напряжений в осаждаемом покрытии. Предложена формула для скорости осаждения покрытия, учитывающая распыление атомов покрытия при произвольных потенциале смещения и угле падения ионов. Показано, что распыление резко снижает скорость осаждения покрытий и делает невозможным осаждение покрытий $\mathrm{TiN}$ и $\mathrm{CrN}$ в режиме постоянного потенциала при потенциалах на подложке, превышающих 1,7 кВ и 0,7 кВ, соответственно, и при нормальном падении ионов. Распыление оказывает наибольшее влияние на внутреннее напряжение и скорость роста покрытия при осаждении ионов под наклонными углами падения $\alpha=45^{\circ} . .70^{\circ}$. Результаты расчетов сравниваются с имеющимися экспериментальными данными.

КЛЮЧЕВЫЕ СЛОВА: плазменно-ионное осаждение покрытий, импульсный потенциал смещения, внутреннее напряжение, нелокальный термоупругий пик иона, скорость осаждения.

\section{ВПЛИВ ПАРАМЕТРІВ ПОТЕНЦАЛУ ЗМЩЩЕННЯ Й ОРІЕНТАЩІЇ ПІДКЛАДКИ НА ХАРАКТЕРИСТИКИ ПОКРИТТЯ, ЩО ОСАДЖУЕТЬСЯ: РОЛЬ РОЗПИЛЕННЯ \\ О.І. Калініченко, О.М. Решетняк, В.С. Стрельницький \\ Національний Науковий Центр «Харківський фізико-технічний інститут» 61108, Харків, Академічна, 1}

Теоретично досліджується вплив процесів атомного розпилення на величину внутрішніх напружень і швидкість росту покриття, одержуваного методом плазмово-іонного осадження 3 використанням імпульсного потенціалу зміщення на підкладці й при різних кутах падіння іонів на поверхню, що осаджується. Формула для розрахунку внутрішніх напружень в покритті, що осаджується, отримана в рамках моделі нелокального термопружного піка іона 3 урахуванням процесів атомного розпилення, використовувалася для розрахунку напружень у покриттях $\mathrm{TiN}$ i CrN, що осаджуються 3 потоків іонів $\mathrm{Ti}^{+} \mathrm{i} \mathrm{Cr}^{+}$, відповідно. Величина напружень для розглянутих покриттів корелює з модулем пружності матеріалу покриття. Максимум кривої напружень зменшується й зміщується в область більш високих потенціалів з ростом кута падіння. Таке поводження обумовлене процесом розпилення квазистабільних міжвузловинних дефектів, що визначають рівень напружень 
в покритті, що осаджується. Запропоновано формулу для швидкості осадження покриття, що враховує розпилення атомів покриття при довільних потенціалі зсуву й куті падіння іонів. Показано, що розпилення різко знижує швидкість осадження покриттів і унеможливлює осадження покриттів $\mathrm{TiN}$ i CrN у режимі постійного потенціалу при потенціалах на підкладці, що перевищують $1,7 \mathrm{\kappa B}$ і $0,7 \mathrm{\kappa B}$, відповідно, й при нормальному падінні іонів. Розпилення впливає на внутрішнє напруження й швидкість росту покриття при осадженні іонів під похилими кутами падіння $\alpha=45^{\circ} . .70^{\circ}$. Результати розрахунків порівнюються з наявними експериментальними даними.

КЛЮЧОВІ СЛОВА: плазмово-іонне осадження покриттів, імпульсний потенціал, внутрішнє напруження, нелокальний термопружний пік іона, скорость осадження.

Процесс вакуумно-дугового осаждения широко используется для модификации поверхности материалов, в том числе для реактивного осаждения на поверхность инструмента и деталей машин упрочняющих покрытий на основе нитридов переходных металлов, которые имеют высокую твердость, прочность, износостойкость, химическую инертность. Поток частиц, генерируемых вакуумной дугой и осаждаемый на подложку, характеризуется высокой степенью ионизации, а при использовании устройств для фильтрации плазмы является полностью ионизированным. Энергия ионов плазмы в процессе осаждения покрытия контролируется приложением потенциала смещения к подложке, что обусловливает возможность гибкого управления структурой и свойствами синтезированных покрытий [1]. Одним из способов повышения характеристик покрытий за счет формирования наноструктуры и улучшения адгезии к подложке является подход, сочетающий процессы осаждения и имплантации. При таком подходе главной особенностью является интенсивная ионная бомбардировка поверхности во время нанесения покрытия, которая обеспечивается путем подачи на подложку высоковольтного импульсного потенциала смещения, что позволяет при низких температурах подложки синтезировать покрытия с плотной нанокристаллической структурой. Изменение параметров импульсного потенциала дает возможность регулировать параметры субструктуры и уровень остаточных напряжений в покрытиях, чрезвычайно высокое значение которых может быть причиной низких эксплуатационных свойств и даже разрушения покрытий [2-6].

Когда говорят о параметрах процесса осаждения, прежде всего, имеют в виду энергию осаждаемых ионов и температуру подложки. В то же время, следует учитывать и ориентацию подложки относительно падающего потока ионов, поскольку, как показывают эксперименты и теория, внутренние напряжения в осаждаемом покрытии, так же как и скорость осаждения, зависят от угла падения ионов [7-11]. Последнее обстоятельство особенно существенно при осаждении покрытия на объекты сложной формы, когда невозможно обеспечить постоянство угла падения ионов на различные участки поверхности осаждения. Прямыми следствиями этого являются неоднородность вдоль поверхности внутренних напряжений и толщины осаждаемого покрытия в случае объектов сложной формы.

Кроме геометрического фактора, определяющего скорость осаждения покрытия и температуру осаждения, заметную роль в формировании покрытия и внутренних напряжений в нем играет распыление частиц покрытия под действием падающих ионов. Влияние распыления может быть настолько существенно, что, при достаточно большой энергии осаждаемых ионов, оно замедляет и даже прекращает рост покрытия. Не менее важным может оказаться влияние распыления и на величину внутренних напряжений, поскольку, наряду с атомами, составляющими кристаллическую структуру покрытия, распыляются также и междоузельные дефекты, отвечающие за внутренние напряжения, что приводит к изменению напряжений. При этом, эффективность распыления может зависеть как от энергии падающих ионов, так и от угла падения ионов на осаждаемую поверхность.

Целью настоящей работы является теоретическое исследование влияния процессов распыления на внутренние напряжения и скорость роста покрытий $\mathrm{TiN}$ и $\mathrm{CrN}$, осаждаемых из потоков ионов $\mathrm{Ti}^{+}$и $\mathrm{Cr}^{+}$, соответственно, в режиме импульсного потенциала при различных углах падения ионов на осаждаемую поверхность.

\section{МАТЕМАТИЧЕСКАЯ МОДЕЛЬ}

В [12] была предложена модель, согласно которой внутренние напряжения формируются в результате генерации напряжений за счет дефектообразования при имплантации ионов и релаксации напряжений в ходе миграции дефектов в точечных тепловых пиках (ТТП) ионов. Полученная в рамках модели формула дала качественное объяснение наблюдающейся зависимости напряжений от энергии ионов и удовлетворительное количественное согласие с результатами экспериментов.

Однако использование модели ТТП для описания релаксации механических напряжений представляется не вполне корректным, поскольку в модели не учитывается характер взаимодействия имплантируемого иона с атомами материала мишени, определяющий начальные размеры и энергосодержание образующегося теплового пика. Вследствие этого модель ТТП принципиально не может объяснить экспериментально наблюдаемую зависимость возникающих внутренних напряжений от температуры осаждения $T_{0}$, a её согласие с экспериментальными данными достигается при величинах энергии активации миграции дефектов $u=3 \ldots 14$ эВ, значительно превосходящих известные значения для процессов миграции междоузельных дефектов. Кроме того, формула, полученная в [12] на основе модели ТТП, не учитывает разнозарядность осаждаемых ионов и режим осаждения покрытия.

В [13-15] предложена модификация формулы для расчёта внутренних напряжений в осаждаемом 
покрытии, основанная на модели нелокального термоупругого пика (НТП) иона- перегретой и перенапряженной области нанометровых размеров, возникающей вокруг траектории иона в материале покрытия в результате термализации фононных потерь иона. Результаты моделирования с помощью программного пакета SRIM 2000 показали, что в случае нормального падения низкоэнергетического иона его НТП может аппроксимироваться сферическим сегментом, примыкающим к поверхности мишени. Основными параметрами НТП являются энергосодержание

$$
E_{p h}=E \eta(E)
$$

и радиус пика

$$
R(t, E)=l(E) / 2+R_{T}(t) .
$$

Здесь $l(E)$ - средний проективный пробег и $\eta(E)$ - доля фононных потерь иона с энергией $E$, вычисляемые с использованием программного пакета SRIM2000 [16], $R_{T}(t)$ - радиус «сферы размывания» точечного теплового источника за время $t$. Центр НТП лежит на середине среднего проективного пробега иона $l$.

Анализ геометрических характеристик каскадов возбужденных атомов, порождаемых ионом при различных энергиях и углах падения, позволяет сделать вывод, что радиус НТП слабо зависит от угла падения $\alpha$. При этом положение НТП относительно границы определяется поворотом НТП на угол $\alpha$ относительно точки влета иона в плоскости, задаваемой нормалью к поверхности мишени и вектором скорости иона.

Объем НТП при произвольном угле падения $\alpha$ определяется выражением:

$$
V(t, E, \alpha)=\frac{4}{3} \pi\left(\frac{l(E)}{2}+R_{T}(t)\right)^{3}-\frac{\pi}{3}\left[\frac{l(E)}{2}(1-\cos \alpha)+R_{T}(t)\right]^{2}\left[l(E)\left(1+\frac{1}{2} \cos \alpha\right)+2 R_{T}(t)\right] .
$$

При увеличении $\alpha$ от $0^{\circ}$ до $90^{\circ}$ объём пика монотонно уменьшается. При нормальном падении $\alpha=0$ выражение (3) совпадает с выражением, приведенным в [13-15].

Что касается энергосодержания пика, то оно в рассматриваемом случае зависит как от энергии иона $E$, так и от угла падения иона $\alpha: E_{p h}(E, \alpha)=E \eta(E, \alpha)$.

Вычисленные функции $V(t, E, \alpha)$ и $E_{p h}(E, \alpha)$ позволяют определить среднюю температуру НТП при наклонном падении пучка ионов

$$
T\left(t, E, T_{0}, \alpha\right)=\frac{E_{p h}(E, \alpha)}{\rho C V(t, E, \alpha)}+T_{0},
$$

где $\rho, C$ и $T_{0}$ - плотность, удельная теплоемкость и температура осаждения покрытия, соответственно. Отметим, что при температурах выше комнатных можно не учитывать зависимость теплоемкости $C$ от температуры, принимая ее равной ее высокотемпературному пределу.

Полученные значения $V(t, E, \alpha)$ и $T\left(t, E, T_{0}, \alpha\right)$ использовались для расчёта скорости релаксации внутренних напряжений в покрытии, определяемой числом термоактивированных переходов $w\left(E, u, T_{0}, \alpha\right)$ в НTП иона:

$$
w\left(E, T_{0}, \alpha\right)=n_{0} v \int_{0}^{\tau_{c}} V(t, E, \alpha) \mathrm{e}^{-\frac{u}{k_{B} T\left(t, E, T_{0}, \alpha\right)}} d t,
$$

где $k_{B}$ - постоянная Больцмана, $n_{0}$ - концентрация атомов мишени, $v$ - частота колебаний атома, $\tau_{c}-$ время жизни НТП, $u$ - энергия активации миграции дефекта.

Внутренние напряжения формируются в результате образования дефектов при имплантации ионов и релаксации напряжений в ходе миграции дефектов в НТП ионов [12-15]. Для определения внутренних напряжений, возникающих в покрытиях при наклонном падении пучка ионов, необходимо вычислить объёмную деформацию мишени. При выводе формулы для напряжений предполагаем, как и в [12-15], линейную зависимость между объемной деформацией мишени и плотностью дефектов, образующихся в результате рассеяния иона на атомах мишени. Результирующая скорость, в расчете на единицу площади, с которой дефекты внедряются в пленку, задается разностью между скоростью появления дефектов за счет имплантации ионов и скоростью их убыли за счет термоактивированной миграции. Вычисления скорости образования дефектов приводят к следующей формуле для расчета внутренних напряжений $\sigma$ в покрытиях, осаждаемых из наклонного потока разнозарядных ионов в режимах постоянного и импульсного потенциала смещения [14]:

$$
\sigma(U, \alpha)=A \frac{E_{Y}}{1-\Pi} \frac{\sum_{i}\left[f t_{p} \chi_{i} \zeta\left(i\left(U+U_{f}+E_{0 i}\right), \alpha\right)+\left(1-f t_{p}\right) \chi_{i} \zeta\left(i\left(U_{1}+U_{f}+E_{0 i}\right), \alpha\right)\right]}{1+\sum_{i}\left[f t_{p} \chi_{i} w\left(i\left(U+U_{f}+E_{0 i}\right), \alpha\right)+\left(1-f t_{p}\right) \chi_{i} w\left(i\left(U_{1}+U_{f}+E_{0 i}\right), \alpha\right)\right]} .
$$

Здесь $E_{Y}$ и П - модуль Юнга и коэффициент Пуассона материала мишени, $t_{\mathrm{p}}$ - длительность прямоугольного 
импульса потенциала с амплитудой $U, f$ - частота следования импульсов, $U_{f}$ - плавающий потенциал, $U_{l}-$ потенциал, подаваемый на подложку между импульсами, $\chi_{i}$ и $E_{0 i}$ - доля ионов с зарядом $i$ (в единицах заряда протона), и начальная энергия иона в расчете на единицу заряда, соответственно. Суммирование проводится по $n$ зарядовым состояниям ионов (как правило, $n \leq 5)$. Функции $\zeta(E, \alpha)$ и $w\left(E, T_{0}, \alpha\right)$, определяющие число стабильных дефектов и скорость их миграции, соответственно, теперь являются также функциями угла падения ионов. Параметр $A$ и значение энергии активации миграции дефектов $u$ определяются из сравнения теоретической зависимости с данными эксперимента при нормальном падении ионов $\alpha=0$. Формула (6) описывает внутренние напряжения, возникающие в покрытиях при осаждении однокомпонентного пучка разнозаряженных ионов в режимах как постоянного $\left(f t_{p}=1\right)$, так и импульсного $\left(f t_{p}<1\right)$ потенциала на подложке.

Температура осаждения $T_{0}$, зависящая как от энергии ионов, так и от угла падения задается соотношением:

$$
T_{0}(U, \alpha)=T_{00}+\frac{\lambda j \cos \alpha}{z}\left[\left(1-f t_{p}\right) \sum_{i} i \chi_{i}\left(U_{1}+U_{f}+E_{0 i}\right)+f t_{p} \sum_{i} i \chi_{i}\left(U+U_{f}+E_{0 i}\right)\right],
$$

где $T_{00}$ - температура необлучаемой подложки, $j$-плотность тока осаждаемых ионов, $z-$ средний заряд иона. Параметр $\lambda$ зависит от конструкционных особенностей установки по осаждению покрытия. Величина $\lambda$ подбирается из условия равенства температуры осаждения ее экспериментальному значению при известном потенциале смещения $U$.

Формула (6) позволила, в рамках модели НТП, объяснить ряд закономерностей, наблюдающихся при осаждении DLC и TiN покрытий в режиме постоянного и импульсного потенциала при нормальном падении ионов фильтрованной вакуумно-дуговой плазмы на осаждаемую поверхность [13-15]. В то же время использование этой формулы для описания внутренних напряжений, возникающих в осаждаемом покрытии при скользящем падении ионов, дает результаты не согласующиеся как с экспериментальными данными, так и с результатами моделирования методами молекулярной динамики [7-10]. Так, расчет по формуле (6) дает увеличение внутренних напряжений во всем диапазоне энергий ионов Ti, используемых при осаждении ТiN покрытия. Причина увеличения расчетных напряжений состоит в том, что при скользящем падении заметно возрастает число дефектов, задаваемых функцией $\zeta(E, \alpha)$, что с избытком компенсирует эффект снижения напряжений за счет более компактного и, в силу этого, более горячего пика. В то же время, в [8] показано, что при осаждении DLC покрытия под наклонными углами $(\alpha \sim \pi / 2)$ в покрытии уменьшается доля $\mathrm{sp}^{3}-$ связей, что косвенно свидетельствует об уменьшении величины внутренних напряжений сжатия. Такой вывод подтверждается результатами моделирования [9], согласно которым внутреннее напряжение в осаждаемом DLC покрытии снижается при изменении $\alpha$ от 0 до $\pi / 4$. Экспериментальные исследования зависимости величины внутренних напряжений в покрытии TiN, осаждаемом как в DC-режиме, так и в режиме импульсного потенциала, также подтверждают уменьшение напряжений при росте угла падения $[7,10]$.

Описанное расхождение теории и эксперимента можно объяснить, если внести определенные коррективы в функцию $\zeta(E, \alpha)$, задающую число стабильных дефектов, создаваемых, в основном, первичным ионом. Для этого необходимо учесть, что некоторая часть дефектов (междоузлий) $\zeta(E, \alpha)$, создающих долговременную деформацию, удаляется из материала мишени в результате распыления. Пусть общее число дефектов, создаваемых как первичным ионом, так и всеми вторичными ионами задается функцией $\zeta_{\text {total }}(E, \alpha)$, а общее число распыленных атомов - функцией $\psi_{\text {total }}(E, \alpha)$. Обе эти функции, так же как и функция $\zeta(E, \alpha)$ могут быть получены с использованием программного пакета SRIM2000 [10]. Поскольку распыляются только атомы из числа $\zeta_{\text {total }}(E, \alpha)$, то часть распыленных стабильных дефектов задается выражением

$$
\psi(E, \alpha)=k \psi_{\text {total }}(E, \alpha) \frac{\zeta(E, \alpha)}{\zeta_{\text {total }}(E, \alpha)},
$$

где $k \sim 1$-параметр, в дальнейших расчетах принимаемый равным единице. В результате число стабильных дефектов, не удаленных из материала в результате распыления, задается выражением

$$
\zeta^{*}(E, \alpha)=\zeta(E, \alpha)-\Delta \zeta(E, \alpha)=\zeta(E, \alpha)\left(1-\frac{\psi_{\text {total }}(E, \alpha)}{\zeta_{\text {total }}(E, \alpha)}\right)
$$

которое должно быть подставлено в (6) вместо функции $\zeta(E, \alpha)$.

С учетом сказанного модифицированная формула для расчета внутренних напряжений в осаждаемом покрытии при произвольном угле падения осаждаемых ионов имеет вид выражения (6), в котором произведена замена $\varsigma(E, \alpha) \rightarrow \varsigma^{*}(E, \alpha)$.

Процессы распыления влияют не только на внутренние напряжения, но они также уменьшают скорость роста покрытия, причем такое уменьшение зависит от угла падения ионов и усиливает эффект чисто 
геометрического фактора (плотность потока ионов при наклонном падении на подложку изменяется по закону «косинусов»). Выражение для скорости роста покрытия в случае импульсного режима осаждения покрытия из пучка разнозарядных ионов можно записать в виде:

$$
v_{d}(U, \alpha)=\frac{M}{\rho} \frac{j}{z} \cos \alpha \sum_{i} \chi_{i}\left\{f t_{p}\left[1-\psi_{\text {total }}\left(i\left(U+U_{f}+E_{0 i}\right), \alpha\right)\right]+\left(1-f t_{p}\right)\left[1-\psi_{\text {total }}\left(i\left(U_{1}+U_{f}+E_{0 i}\right), \alpha\right)\right]\right\},(1
$$

где $M$ и $\rho$ - масса молекулы и плотность осаждаемого покрытия, соответственно, $z$ - средний заряд иона. Поскольку азот попадает в покрытие вследствие хемосорбции, то при выводе (10) предполагалось, что каждый ион металла, осаждаемый на подложку, приводит к образованию одной «молекулы» нитрида в покрытии.

\section{РЕЗУЛЬТАТЫ И ИХ ОБСУЖДЕНИЕ}

Полученные формулы позволяют рассчитывать внутренние напряжения и скорости осаждения покрытий, осаждаемых из потоков разнозарядных ионов в режимах постоянного и импульсного потенциалов и при различных углах падения ионов (рис. 1,2).

Расчет внутренних напряжений в TiN покрытии, осаждаемом из пучка ионов $\mathrm{Ti}^{+}$, проводился с использованием формулы (6) при следующих значениях параметров: $u=0,58$ эВ, $U_{f}=20 \mathrm{~B}, t_{p}=5$ мкс, $f=24$ кГц, $T_{00}=300 \mathrm{~K}$. Параметры НТП ионов $\mathrm{Ti}^{+}$в материале покрытия TiN, необходимые для вычисления функций $\zeta^{*}(E)$ и $w(E)$, определялись с использованием программного пакета SRIM2000. При расчётах также принималось $\mu j=0,186 \mathrm{~K} / \mathrm{B}$, что соответствовало повышению температуры подложки на $\sim 40 \mathrm{~K}$ при увеличении потенциала на 1 кВ в режиме импульсного потенциала с выбранными значениями параметров $f$ и $t_{p}$. Экспериментальное значение напряжения $\sigma=10,6 \mathrm{GPa}$ при потенциале смещения $U=1,5 \mathrm{kV}$, приведенное в [13] использовалось для определения константы $A$ в выражении (6).

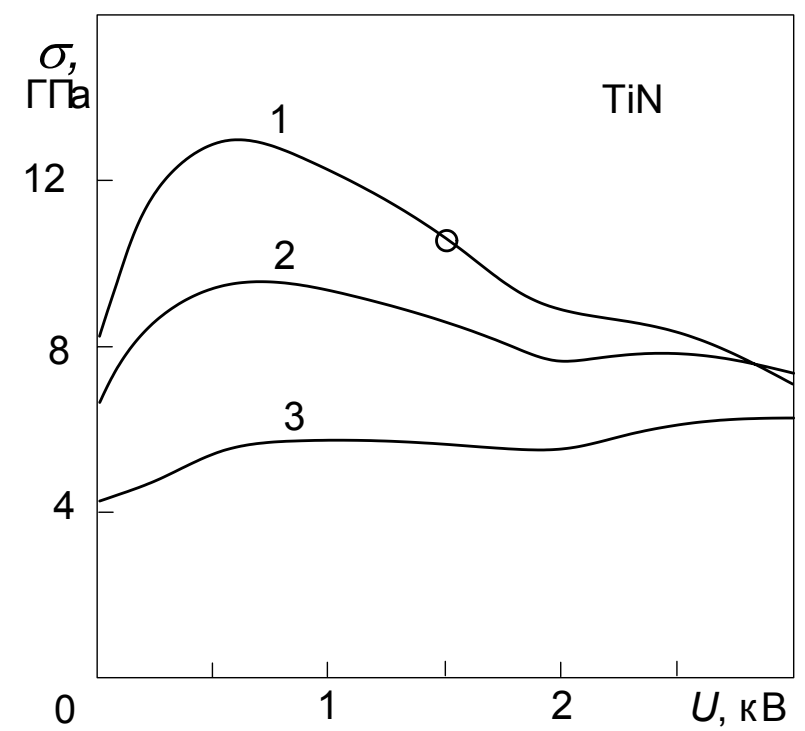

Рис. 1. Внутренние напряжения в покрытии TiN, осаждаемом из потока ионов $\mathrm{Ti}^{+}$в импульсном режиме при углах падения $\alpha=0^{\circ}, 45^{\circ}, 70^{\circ}$ (кривые $1-3$, соответственно).

Светлый кружок - экспериментально определенное значение напряжений в покрытии $\mathrm{TiN}$ при потенциале $U=$ $1,5 \mathrm{kV}$ и нормальном падении ионов [10].

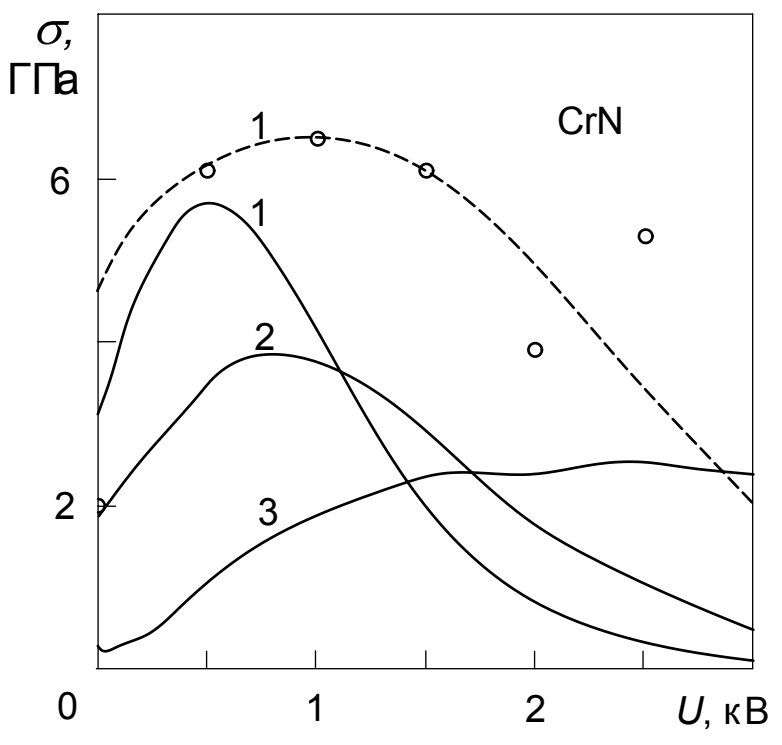

Рис. 2. Внутренние напряжения в покрытии $\mathrm{CrN}$, осаждаемом из потока ионов $\mathrm{Cr}^{+}$в импульсном режиме при углах падения $\alpha=0^{\circ}, 45^{\circ}, 70^{\circ}$ (кривые 1-3, соответственно). Пунктирная кривая - расчетные напряжения в покрытии $\mathrm{Cr}_{0.5} \mathrm{Al}_{0.5} \mathrm{~N}$, светлые кружки данные эксперимента [17] при нормальном падении ионов.

Расчет внутренних напряжений в $\mathrm{CrN}$ покрытии, осаждаемом из пучка ионов $\mathrm{Cr}^{+}$, также проводился с использованием формулы (6) при следующих значениях параметров: $u=0,75$ эВ, $U_{f}=20 \mathrm{~B}, t_{p}=12$ мкс, $f=$ 12 кГц, $T_{00}=300 \mathrm{~K}$. Параметры НТП ионов $\mathrm{Cr}^{+}$в материале покрытия $\mathrm{CrN}$ также определялись с использованием программного пакета SRIM2000. При расчётах также принималось $\mu j=0,3 \mathrm{~K} / \mathrm{B}$, что соответствовало повышению температуры подложки на $70 \mathrm{~K}$ при увеличении потенциала на 1 кВ в режиме импульсного потенциала с выбранными значениями параметров $f$ и $t_{p}$. Температура осаждения при нулевом потенциале смещения составляла $T_{0}(0)=370 \mathrm{~K}$. Для определения константы $A$, задающей масштаб напряжений в покрытии $\mathrm{CrN}$ использовались экспериментальные данные, полученные для покрытия $\mathrm{Cr}_{0.5} \mathrm{Al}_{0.5} \mathrm{~N}$ [17]. Значения параметров $\chi_{i}$ и $E_{0 i}$ для ионов $\mathrm{Ti}^{+}$и $\mathrm{Cr}^{+}$брались из монографии [1]. 
На рис. 1 представлены результаты расчетов внутренних напряжений в зависимости от потенциала смещения в покрытии $\mathrm{TiN}$, осаждаемом из потока ионов $\mathrm{Ti}^{+}$при различных углах падения ионов. Из рисунка следует, что максимум кривой напряжений при нормальном падении составляет 13 ГПа, что значительно превышает величину ( 10 ГПа), приводимую ранее [14]. Отмеченное различие объясняется тем, что в [14] приведены данные по напряжениям в покрытии $\mathrm{TiN}$, осаждаемом из более плотного потока ионов $\mathrm{Ti}^{+}$. Это приводило к значительно большей температуре подложки ( $\mu j=0,3 \mathrm{~K} / \mathrm{B})$ и, как следствие, к меньшим напряжениям в осаждаемом покрытии. Максимум кривой напряжений уменьшается и смещается в область более высоких потенциалов с ростом угла падения. Указанное поведение внутренних напряжений всецело обусловлено процессом распыления, затрагивающим, как отмечалось выше, и междоузельные дефекты, определяющие уровень напряжений в покрытии. Как показывает проведенный анализ, в отсутствие эффекта распыления дефектов внутренние напряжения увеличивались бы с ростом угла падения ионов в силу ускорения дефектообразования при наклонных углах падения, что вступало бы в противоречие с имеющимися экспериментальными данными [7,10]. Наибольшее влияние процессов атомного распыления на формирование внутренних напряжений имеет место при осаждении ионов под наклонными углами падения $\alpha=45^{\circ} . .70^{\circ}$.

Аналогичное поведение демонстрируют напряжения в покрытии $\mathrm{CrN}$, осаждаемом из потока ионов $\mathrm{Cr}^{+}$ (см. рис. 2). Отметим, что величина напряжений в исследованных покрытиях коррелирует с модулем упругости материала покрытия. При больших углах падения зависимость величины напряжений от потенциала на подложке ослабевает для обоих исследованных материалов.

Рассмотрим влияние угла падения ионов на скорость осаждения покрытия. Моделирование с помощью программного пакета SRIM2000 показывает, что количество распыленных атомов с ростом энергии ионов увеличивается значительно сильнее для скользящего падения $\left(\alpha>0^{\circ}\right)$ по сравнению с нормальным падением. Соответственно, уменьшается поток осаждаемых атомов и скорость роста покрытия.

На рис. 3,4 представлены результаты расчетов коэффициента распыления, задаваемого выражением

$$
K(U, \alpha)=\sum_{i} \chi_{i}\left[f t_{p} \psi_{\text {total }}\left(i\left(U+U_{f}+E_{0 i}\right), \alpha\right)+\left(1-f t_{p}\right) \psi_{\text {total }}\left(i\left(U_{1}+U_{f}+E_{0 i}\right), \alpha\right)\right]
$$

атомов $\mathrm{Ti}(\mathrm{Cr})$ при падении потока ионов $\mathrm{Ti}^{+}\left(\mathrm{Cr}^{+}\right)$на покрытие $\mathrm{TiN}(\mathrm{CrN})$, осаждаемое в режиме импульсного потенциала при различных углах падения ионов. Рассматривается режим импульсного потенциала с коэффициентом заполнения цикла $f t_{p}=0,12\left(f t_{p}=0,144\right)$. Пунктирные кривые соответствуют коэффициентам распыления при тех же углах падения ионов, но в случае режима постоянного потенциала.

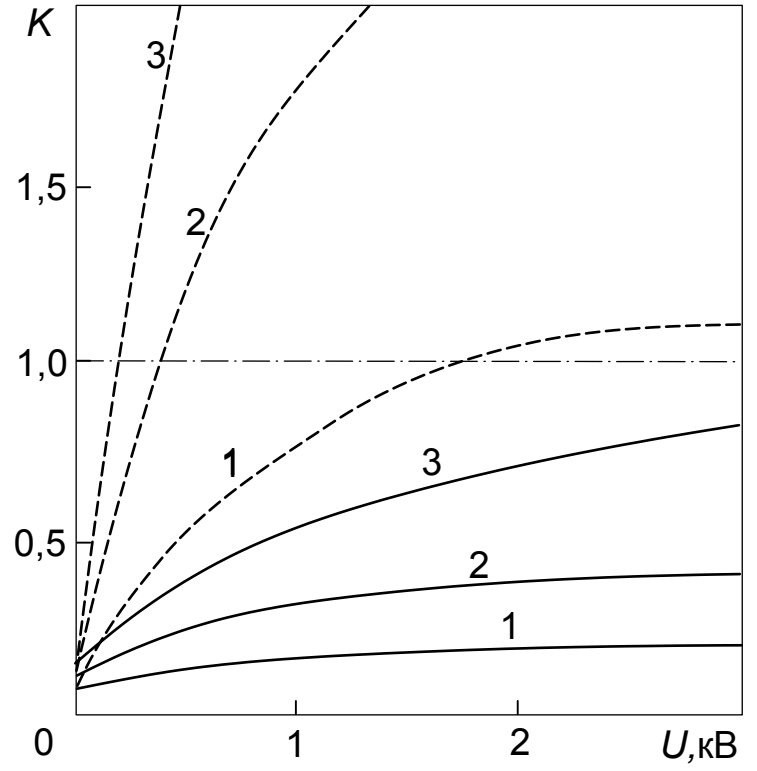

Рис. 3. Коэффициенты распыления $K(U)$ атомов Ті при падении потока ионов $\mathrm{Ti}^{+}$на покрытие $\mathrm{TiN}$, осаждаемое в режиме импульсного потенциала при углах падения $\alpha=0^{\circ}, 45^{\circ}, 70^{\circ}$ (сплошные кривые 1 - 3 , соответственно). Пунктирные кривые - соответствующие коэффициенты распыления в случае режима постоянного потенциала

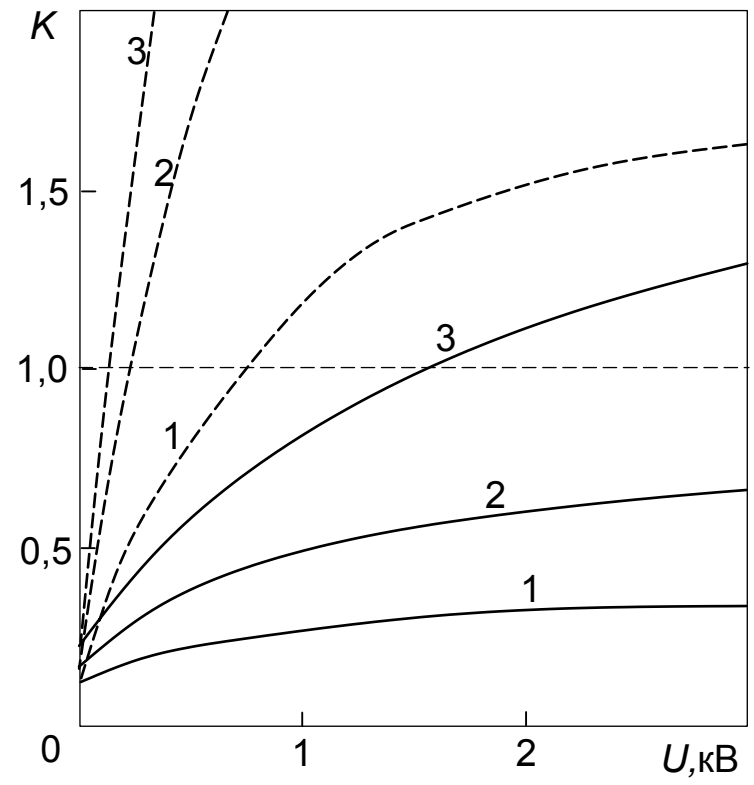

Рис. 4. Коэффициенты распыления $K(U)$ атомов $\mathrm{Cr}$ при падении потока ионов $\mathrm{Cr}^{+}$на покрытие $\mathrm{CrN}$, осаждаемое в режиме импульсного потенциала при углах падения $\alpha=0^{\circ}, 45^{\circ}, 70^{\circ}$ (сплошные кривые 1 - 3 , соответственно). Пунктирные кривые - соответствующие коэффициенты распыления в случае режима постоянного потенциала

Из рис. 3 видно, что в случае осаждения покрытия TiN в режиме постоянного потенциала при нормальном падении ионов $\mathrm{Ti}^{+}$коэффициент распыления атомов Ті достигает 1 при потенциале на подложке $U=1,7$ кВ, то 
есть, пленка перестает осаждаться, а при дальнейшем возрастании потенциала количество распыленных атомов превышает количество осаждаемых ионов: происходит травление поверхности покрытия ТiN. Такой переход от режима осаждения к режиму травления хорошо согласуется с литературными данными [1]. По мере увеличения угла падения в интервале от $0^{\circ}$ до $70^{\circ}$ значение коэффициента распыления сильно возрастает. Переходу от распыления к травлению соответствуют значительно меньшие потенциалы, а именно: 400 В и 190 В для углов падения $45^{\circ}$ и $70^{\circ}$, соответственно. При осаждении в режиме импульсного потенциала значение коэффициента распыления значительно ниже, чем в режиме постоянного потенциала и существенно зависит от коэффициента заполнения цикла $f t_{p}$. Из сравнения рис. 3,4 видно, что процессы распыления при осаждении покрытия CrN играют более существенную роль, чем при осаждении покрытия TiN, что связано с большим атомным весом ионов $\mathrm{Cr}^{+}$. Так, при нормальном падении в режиме постоянного потенциала процесс осаждения покрытия $\mathrm{CrN}$ сменяется на процесс травления уже при потенциале 0,7 кВ. Для углов падения $45^{\circ}$ и $70^{\circ}$ расчет дает значения потенциалов 230 В и 130 В, соответственно.

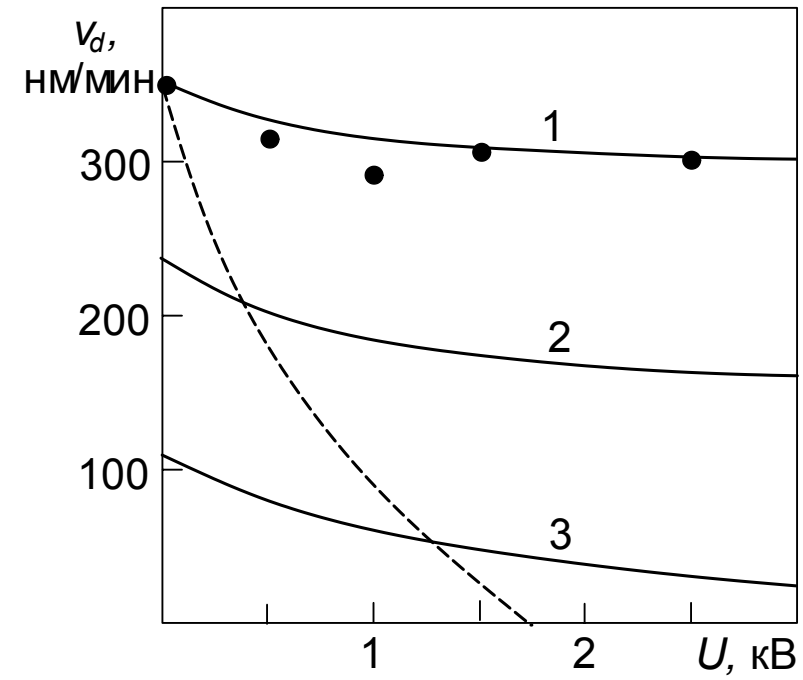

Рис. 5. Зависимость скорости роста покрытия TiN от потенциала смещения на подложке при углах падения ионов $\mathrm{Ti}^{+} \alpha=0^{\circ}, 45^{\circ}, 70^{\circ}$ (кривые $1,2,3$, соответственно). Черные кружки - данные эксперимента [18]. Пунктирная кривая соответствует скорости роста при постоянном потенциале и нормальном падении ионов.

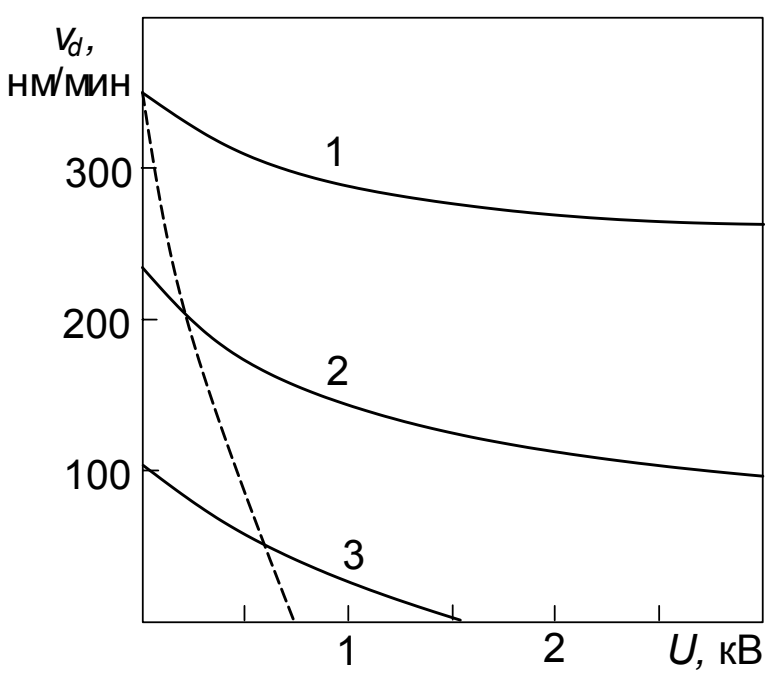

Рис. 6. Зависимость скорости роста покрытия $\mathrm{CrN}$ от потенциала смещения на подложке при углах падения ионов $\mathrm{Cr}^{+} \alpha=0^{\circ}, 45^{\circ}, 70^{\circ}$ (кривые $1,2,3$, соответственно). Пунктирная кривая соответствует скорости роста при постоянном потенциале и нормальном падении ионов.

На рис. 5,6 представлена зависимость скорости роста покрытия $\mathrm{TiN}(\mathrm{CrN})$ от потенциала смещения на подложке при различных углах падения ионов $\mathrm{Ti}^{+}\left(\mathrm{Cr}^{+}\right)$на осаждаемую поверхность. Рассматривался режим импульсного потенциала с коэффициентом заполнения цикла $f t_{p}=0,12\left(f t_{p}=0,144\right)$. Расчет проводился в предположении, что эффект уплотнения обеспечивает плотность покрытия, совпадающую с табличным значением массивного материала [19]. Для сравнения приведена кривая скорости роста при постоянном потенциале и нормальном падении ионов (пунктирная кривая). Из рис. 5 видно, что имеет место качественное согласие расчетной кривой скорости осаждения с экспериментальными данными, полученными для случая осаждения покрытия TiN при нормальном падении ионов $\mathrm{Ti}^{+}$. Также видно, что скорость осаждения покрытия в импульсном режиме при нормальном падении ионов слабо (на $\sim 13 \%$ ) уменьшается при увеличении потенциала смещения на подложке в диапазоне $0 . .3$ кВ. С увеличением угла падения ионов скорость осаждения уменьшается, и ее падение с ростом потенциала существенно возрастает - до $33 \%$ и $79 \%$ для углов падения $45^{\circ}$ и $70^{\circ}$, соответственно.

\section{ВЫВОДЫ}

1. Формула для расчета внутренних напряжений в осаждаемом покрытии при произвольном угле падения ионов, полученная в рамках модели нелокального термоупругого пика иона с учетом процессов атомного распыления, использовалась для расчета напряжений в покрытиях $\mathrm{TiN}$ и $\mathrm{CrN}$. Исследование влияния процессов распыления на формирование внутренних напряжений показало, что такое влияние наиболее существенно при осаждении ионов под наклонными углами падения $\alpha=45^{\circ} . .70^{\circ}$.

2. Величина напряжений для рассмотренных покрытий коррелирует с модулем упругости материала покрытия. Максимум кривой напряжений уменьшается и смещается в область более высоких потенциалов с 
ростом угла падения в соответствии с имеющимися экспериментальными данными. Указанное поведение обусловлено процессом распыления междоузельных дефектов, определяющих уровень напряжений в осаждаемом покрытии.

3. Предложена формула для скорости осаждения покрытия, учитывающая распыление атомов покрытия при произвольных потенциале и угле падения ионов. Сравнение расчетной кривой скорости осаждения TiN покрытия при нормальном падении ионов $\mathrm{Ti}^{+}$с данными эксперимента показало их качественное согласие.

4. Анализ показал, что процесс атомного распыления резко снижает скорость осаждения покрытий в режиме постоянного потенциала уже при потенциалах $0,1 \ldots 1$ кВ и делает невозможным осаждение покрытий $\mathrm{TiN}$ и $\mathrm{CrN}$ при потенциалах на подложке, превышающих 1,6 кВ и 0,7 кB, соответственно, и при нормальном падении ионов, что согласуется с экспериментальными данными. При увеличении угла падения потенциал, при котором процесс осаждения сменяется процессом травления, существенно снижается.

\section{СПИСОК ЛИТЕРАТУРЫ}

1. Aksenov I.I., Andreev A.A., Belous V.A. et al. Vakuumnaja duga: istochniki plazmy, osazhdenija pokrytij, poverhnostnoe modificirovanie [Vacuum arc: sources of plasma, deposition of coatings, surface modification]. - Kyyiv: Naukova dumka, 2012. - 727p. (In Russian)

2. . Bilek M.M, McKenzie D.R., Tarrant R.N., Lim S.H.M., McCulloch D.G. Plasma-based ion implantation utilising a cathodic arc plasma // Surf. Coat. Technol. 2002. - Vol. 156. - P. 136-142.

3. Pelletier J., Anders A. Plasma-based ion implantation and deposition: A review of physics, technology, and applications // IEEE Transactions on Plasma Science. - 2005. - Vol. 33. - P. 1944-1959.

4. Akkaya S.S., Vasyliev V.V., Reshetnyak E.N., Kazmanlı K., Solak N., Strel'nitskij V.E., Urgen M. Structure and properties of TiN coatings produced with PIII\&D technique using high efficiency rectilinear filter cathodic arc plasma // Surface \& Coatings Technology. - 2013. - Vol.236 - P. 332-340.

5. Belous V., Vasyliev V., Luchaninov A., Marinin V., Reshetnyak E., , Strel'nitskij V., Goltvyanytsya S., Goltvyanytsya V. Cavitation and abrasion resistance of Ti-Al-Y-N coatings prepared by the PIII\&D technique from filtered vacuum-arc plasma // Surface \& Coatings Technology. - 2013. - Vol. 223. - P. 68-74.

6. Vasyliev V., Goltvyanytsya S., Goltvyanytsya V., Marinin V., Reshetnyak E., Strel'nitskij V., Tolmacheva G. Durability of the multicomponent nitride coatings based on TiN and (Ti,Al)N deposited by PIII\&D method. // Problems of Atomic Science and Technology. - 2015. - No.2. - P. 130-138.

7. Jiang F., Zhang T.F., Wu B.H., Yu Y., Wu Y.P., Zhu Sh.F., Jing F.J., Huang N., Leng Y.X. Structure, mechanical and corrosion properties of TiN films deposited on stainless steel substrates with different inclination angles by DCMS and HPPMS // Surface \& Coatings Technology. - 2016. - Vol.29, - P. 54-62.

8. Nelson N., Rakowski R.T., Franks J., Woolliams P., Weaver P., Jones B.J. The effect of substrate geometry and surface orientation on the film structure of DLC deposited using PECVD // Surface and Coatings Technology. - 2014. - Vol.254. P. 73-78.

9. Xiaowei Li, Peiling Ke, Kwang-Ryeol Lee, AiyingWang Molecular dynamics simulation for the influence of incident angles of energetic carbon atoms on the structure and properties of diamond-like carbon films // Thin Solid Films. - 2014. - Vol.552. P. 136-140.

10. Vasil'ev V.V., Luchaninov A.A., Reshetnyak E.N., Strelnitskij V.E. Effect of substrate position relative to the flow of filtered cathodic-arc plasma on the structure and properties of TiN coatings // Problems of Atomic Science and Technology. - 2017. No 2. - P.160-167.

11. Kalinichenko A.I., Perepelkin S.S., Strel'nitskij V.E. Influence of incident angle of ion beam on intrinsic stress in deposited coating // Journal of Surface Physics and Engineering. - 2016. - Vol. 1. - No. 12. - P. 338-345. (In Russian).

12. Davis C.A. A simple model for the formation of compressive stress in thin films by ion bombardment // Thin Solid Films. 1993. - Vol. 226. - No. 2-3. - P. 30-34.

13. Kalinichenko A.I., Perepelkin S.S., Strel'nitskij V.E. Dependence of intrinsic stress and structure of ta-C film on ion energy and substrate temperature in model of the non-local thermoelastic peak // Diamond Relat. Mater. - 2010. - Vol. 19. - P. 996-998.

14. Kalinichenko A.I., Kozionov S.A., Perepelkin S.S., Strel'nitskij V.E. Intrinsic stresses in coatings deposited at plasma immersion ion implantation // East European Journal of Physics. - 2014. - Vol. 1. - No. 4. - P. 58-64. (In Russian).

15. Kalinichenko A.I., Perepelkin S.S., Strel'nitskij V.E. Intrinsic stresses in CrN coatings deposited at different angles of incidence ions // Proceedings of the 2017 IEEE 7th International conference on Nanomaterials: Application \& Properties (NAP2017), Zatoka, Ukraine - 2017. - Part 1. - 01PCS104(4p.).

16. Ziegler J.F., Biersack J.P., Littmark U. The Stopping and Range of Ions in Solids. - New York: Pergamon Press, 1996. - 297 p.

17. Vasyliev V.V., Luchaninov A.A., Reshetnyak E.N., Strel'nitskij V.E., Tolmacheva G.N., Pribytkov G.A., Korzhova V.V. Structure and properties of nitride coatings deposited from filtered vacuum arc plasma generated by evaporation of cromiumaluminum powder cathode // Journal of Surface Physics and Engineering. - 2016. - Vol. 1. - No. 1. - P. 62-80.

18. Vasyliev V.V., Luchaninov A.A., Reshetnyak E.N., Tolmacheva G.N., Strel'nitskij V.E. Vliyanie impulsnogo potentsiala smescheniya na strukturu i tverdost TiN pokrytiy, osazhdennyih iz filtrovannoy vakuumno-dugovoy plazmyi // Sbornik trudov 4 Mezhdunarodnoy nauchnoy konferentsii "Fiziko-himicheskie osnovyi formirovaniya i modifikatsii mikro- i nanostruktur", Kharkov, Ukraina. - 2010. - Vol. 1. - P. 85 - 89.

19. Bakai A.S., Sleptsov S.N., Zhukov A.I., Marchenko I.G., Sleptsov A.N. Mathematical Modelling of the Densification of Niobium Film Deposited from Self-Ion-Atomic Fluxes // Met. Phys. Adv. Tech.- 1996.- Vol. 15.- P. 1329-1342. 